\title{
Perceived barriers to early progressive mobilization in the ICU: Multidisciplinary perspectives in an underserved population
}

\author{
Jessica Grimm*1, Angela Silvestri-Elmore ${ }^{2}$, Erik Grimm ${ }^{1}$, Kelly Klinger ${ }^{3}$, Scott Nye ${ }^{3}$, Jessica Bhullar ${ }^{1}$ \\ ${ }^{1}$ School of Nursing, Touro University Nevada, Henderson, NV, USA \\ ${ }^{2}$ School of Nursing, University of Nevada, Las Vegas, NV, USA \\ ${ }^{3}$ School of Physical Therapy, Touro University Nevada, Henderson, NV, USA
}

Received: December 6, 2018

DOI: $10.5430 /$ jnep.v9n5p102
Accepted: January 16, 2019

Online Published: January 25, 2019

\begin{abstract}
Early progressive mobilization is the initiation of movement when a patient is hemodynamically stable, adequately oxygenated, and minimally able to participate. Early progressive mobilization has been linked to decreased morbidity and mortality as inactivity has a profound adverse effect on the brain, skin, skeletal muscle, pulmonary and cardiovascular systems. Literature supports early progressive mobilization and physical therapy as a safe and effective intervention that can have a positive impact on functional outcomes. While the benefits of early progressive mobilization in the intensive care unit have been well documented in recent years, many intensive care units are unable to effectively integrate early progressive mobilization into their daily practice. Therefore, the purpose of this project was to determine whether an educational intervention on the Early Progressive Mobilization Protocol at an urban intensive care unit in Las Vegas, Nevada affected knowledge of, skill in, and attitudes toward implementation of the protocol in practice, as well as to determine whether there was a difference in reported compliance scores among various disciplines. A pre-test survey designed to examine these variables and the reported compliance with the Early Progressive Mobilization protocol was administered. Educational sessions were provided to participants on the Early Progressive Mobilization Protocol after the pre-test. A post-test was administered after the educational session to determine the educational impact on the identified variables. Data analysis was completed using frequency distributions. Valuable insight was gained on the potential impact of targeted educational intervention. Further study is warranted to assess the effects of routine training in intensive care units with similar protocols.
\end{abstract}

Key Words: Early progressive mobilization, Barriers, Addressing barriers, Quality improvement

\section{INTRODUCTION}

EPM is the initiation of movement when a patient is hemodynamically stable, adequately oxygenated, and minimally able to participate in care. EPM has been linked to decreased morbidity and mortality as inactivity has a profound adverse effect on the brain, skin, skeletal muscle, pulmonary and cardiovascular systems. ${ }^{[1]}$ Literature supports EPM and physical therapy (PT) as a safe and effective intervention that can have a positive impact on functional outcomes. ${ }^{[2]}$ While the benefits of EPM in the intensive care unit (ICU) have been well documented in recent years, many ICUs are unable to effectively integrate EPM into their daily practice. In 2018, the Society of Critical Care Medicine (SCCM) published new Clinical Practice Guidelines for the Prevention and Management USA. 
of Pain, Agitation/Sedation, Delirium, Immobility, and Sleep Disruption (PADIS) for Adult Patient. The PADIS Guidelines are designed to serve as a roadmap for optimizing ICU patient care experiences and improve patient outcomes. ${ }^{[3]}$ Included in these guidelines is the ABCDEF Bundle which designed to reduce delirium, improve pain/sedation management, and overall reduce harm to patients in the short and long term after critical illness.

"A" stands for "assess, prevent and manage pain", "B" stands for "Both Spontaneous awakening trials and Spontaneous breathing trials", "C" stands for "choice of analgesia and sedation", "D" stands for "Delirium: Assess, prevent and manage", "E" stands for "early mobility and exercise", and "F" stands for "Family Engagement and Employment". ${ }^{[3]}$ The "E" component of ABDCEF Bundle is highly relevant to this project and addresses the issue of early mobility and exercise. ${ }^{[3]}$ This topic has been established as one that can significantly impact on patient outcomes. Mobilizing patients out of bed is frequently delayed due to competing priorities in busy critical care units and varying levels of RN' knowledge and motivation. ${ }^{11]}$ Additional barriers to EPM in the ICU setting include equipment, staffing and time management issues. ${ }^{[4]}$ Examination of barriers can serve to guide practice changes and educational approaches regarding implementing early progressive mobilization protocols. ${ }^{[4]}$

\subsection{Background}

EPM in ventilated patients is an emerging topic in contemporary critical care practice. ${ }^{[5-8]}$ Ventilating using positive pressure came about originally in the 1940's to improve oxygen levels for pilots while at very high altitudes. It wasn't until the 1960's that it found a place in medicine and it took until the 1970's to become popular in the medical field. Ventilators have allowed for the ability to prolong life of critically ill patients. Until recently it was common for patients to be sedated and left on a ventilator for long periods of time. It was thought that by keeping the patient sedated they could rest, have less anxiety, and thus heal more quickly. Mobilization of ventilated patients generally consisted of passive range of motion and repositioning in bed. Current findings have brought deeper understanding to the effects that come from prolonged sedation and mechanical ventilation. Ventilators in combination with sedation have presented many new challenges such as muscle deconditioning, functional decline, delirium, hospital acquired infections, along with other long-term effects on cognition and mortality. ${ }^{[9]}$

\subsection{Problem statement}

In 2018, the SCCM updated recommendations for optimization of critical care practice to include recommendations for

Published by Sciedu Press
EPM and appropriate progressive rehabilitation services for ICU patients. ${ }^{[3]}$ Currently, at the host site which is an urban 293-bed hospital in Las Vegas, Nevada, anecdotal staff reports show that mobilization of ventilated patients consists of turning patients every two hours, pulling patients up in bed as needed, and transferring patients to a stretcher for necessary testing. PT is not consistently ordered until after extubating, omitting the patient's opportunity to reap the benefits of EPM. Multidisciplinary compliance is a necessary component of a successful EPM protocol in the ICU setting. In 2014, this project's host site implemented an EPM protocol for the ICU setting. This nurse driven protocol has been correlated with staff reports of difficult implementation and poor compliance from the multidisciplinary team. Studies have shown that best practice necessitates EPM of ventilated patients for optimal patient outcomes. ${ }^{[10-13]}$ Understanding barriers to implementation in terms of knowledge, skills, and attitudes may help to tailor future efforts in EPM and protocol compliance. ${ }^{[14]}$

\subsection{Project aims}

The purpose of this project was to:

(1) Develop an education module addressing potential barriers to compliance of the ICU EPM protocol.

(2) Disseminate an education module on EPM focused on barriers to implementation to the multidisciplinary team.

(3) Examine the effects of education on perceptions surrounding EPM.

(4) Examine the effects of education on reported compliance among identified disciplines.

(5) Promote patient safety and quality patient care through education on ways to overcome barriers to EPM.

\subsection{Project questions}

The questions guiding this project were as follows:

(1) Will an educational intervention on the EPM protocol increase the knowledge level of the protocol at the project site?

(2) Will an educational intervention on the EPM protocol increase reported skill in implementing the protocol at the project site?

(3) Will an educational intervention on the EPM protocol improve the attitudes towards the protocol at the project site?

(4) Is there a difference in reported likelihood to comply with the EPM among various disciplines (nursing, PT, occupational therapy [OT], respiratory therapy $[\mathrm{RT}]$, licensed provider)? 


\subsection{Search terms}

Electronic databases were accessed to perform a comprehensive literature review, including Cumulative Index to Nursing and Allied Health Literature (CINAHL), Medline, PubMed, EBSCO, Clinical Key, UpToDate, and Cochrane Library. The selected key search terms included "ICU early mobility", "ICU mobility barriers", "Implementing ICU early progressive mobility protocol". Search filters included studies on humans, and the English language. Inclusion criteria included literature published from the year 2008 to 2018, systematic reviews, non-randomized and randomized trials, and comparative studies. Studies published prior to 2008 were considered for historical relevance. Exclusion criteria included studies not relevant to the acute care environment. Of the articles returned, 38 articles were selected for inclusion.

\section{REVIEW OF THE RELATED LITERATURE}

Immobility during critical illness has been associated with post-intensive care syndrome (PICS) which includes abnormalities in cognition, mental health, and physical function following critical care illness. ${ }^{[2]}$ ICU-acquired weakness is the most prominent of physical function abnormalities identified with an incidence rate of $25 \%$ or more of post-ICU patients. ${ }^{[15,16]}$ ICU-acquired weakness has been strongly associated with prolonged ventilation (greater than 7 days), sepsis, and multi-system organ failure. ${ }^{[2]}$ Although some causes of weakness in ICU patients can be associated with other problems such as pre-existing illnesses, muscle weakness disorders, or other organic causes of weakness; many cases of ICU acquired weakness are preventable and responsive to treatment. ${ }^{[2]}$ In a multicenter study, at 6 months after discharge from ICU care, $64 \%$ of patients reported continued impairment in mobility function. ${ }^{[17]}$ Many of these patients reported needing additional assistance with daily care. This dynamic poses a financial burden on the patient and their family and negatively affects overall quality of life. ${ }^{[17]}$ EPM has been established as a national standard of care for ICU patients and is considered a safe and effective intervention that can have a positive impact on functional outcomes. ${ }^{[18]}$ EPM has been shown to be associated with positive patient outcomes including decreased ICU acquired weakness, greater unassisted walking distance at discharge, and improved quality of life after discharge. ${ }^{[3]}$ The SCCM published a National Advisory Statement in 2014 showing the first national push toward EPM as a standardized practice. ${ }^{[3]}$ The PADIS Guidelines established by SCCM in 2018 provided further clarity on the practice of EPM through the ABCDEF bundle. ${ }^{[3]}$ The "E" component provides very specific direction on EPM and exercise including assessing appropriateness for EPM.

The SCCM has illustrated the benefit of EPM of ICU patients 104 in improving muscle strength, functional mobility, quality of life, decreasing overall cost as well as length of stay and duration of mechanical ventilation. ${ }^{[8,10,19,21]}$ Establishment of a standard of care using evidence based practice may assist the inexperienced practitioners to improve the overall quality of patient care. Development of evidence based protocols focused on current national guidelines of care is important to facilitate improvement in quality of care within this environment. ${ }^{[1,6,10-13,22,23]}$ Despite the availability of national guidelines and the presence of protocols to guide EPM in ICU's, many healthcare organizations continue to face barriers in successful implementation of EPM. ${ }^{[8]}$ Identifying barriers to EPM can aid in addressing the barriers and ultimately lead to solutions that will have a positive impact on patient outcomes. ${ }^{[8]}$ The major themes from the literature that were identified as pertinent to this project site include benefits of EPM and evidence based approaches to addressing barriers of EPM.

\subsection{Benefits of EPM}

EPM has demonstrated many benefits across the literature including reduction in healthcare costs, improved functional status at discharge and overall minimizing negative effects of critical illness. ${ }^{[24]}$ One of the most well studied benefits is that of improved functional mobility at discharge. ${ }^{[8,25]}$ Patients who are initiated with EPM in the first 72 hours of critical illness experience improved physical function on discharge. ${ }^{[7]}$ In an international multicenter randomized control trial, 200 ICU's compared care approaches of early goal directed mobilization to usual care and found that EPM improved objective mobilization of the patients and had a significant impact in reducing length of stay. ${ }^{[26]}$ This project did note that $70 \%$ of their patients did not meet inclusion criteria of their protocols which demonstrated limitations for application. ${ }^{[26]}$ Several small-scale randomized control trials have compared EPM to usual care and found that EPM is safe and is associated with decreased duration of delirium, fewer ventilator days, and improved functional mobility at the time of discharge. ${ }^{[13,27]}$ A meta-analysis of four trials further demonstrated that when comparing EPM to usual care, patients who received EPM also experienced shorter ventilator duration and improved functional mobility at discharge. ${ }^{\text {[9] }}$ In another meta-analysis, researchers examined the benefits to successful EPM programs. Key factors that emerged in their discussion included improved muscle strength, physical function, quality of life at discharge, reduced duration of mechanical ventilation, length of stay, and cost. ${ }^{[8]}$ EPM decreases overall cost of care. ${ }^{[8]}$ The average cost of a day in the ICU is $\$ 3,184 .{ }^{[8]}$ EPM has been shown to decrease overall hospital length of stay by 3.1 days with a national average of $\$ 2,157$ per day beyond the second day. ${ }^{[8]}$ The ben- 
efits of EPM have been well established in the literature and demonstrate that EPM is a safe and effective care approach for critically ill patients. ${ }^{[2]}$

\subsection{Examining the barriers}

Barriers to EPM have been well identified across current literature. Identifying barriers to EPM can aid in addressing the barriers early on. ${ }^{[28]}$ Barriers to EPM may include inadequate multidisciplinary staffing, difficulties with collaboration among disciplines, sedation, poor staff knowledge around EPM. ${ }^{[4,29-32]}$ Another group of researchers provided a comprehensive list of barriers from 3 different hospitals and provided possible solutions to the barriers to promote mobility. ${ }^{[14]}$ Barriers to EPM identified in this project include the following: lack of leadership, lack of staffing and equipment, lack of knowledge and training, lack of physician referrals for PT, over-sedation, delirium, patient hemodynamic tolerance of activity, and safety concerns. ${ }^{[14]}$ Of these potential barriers, administrators at this project site have identified lack of dedicated leadership for EPM, lack of staffing and equipment, lack of physician referrals for PT, over-sedation, and lack of knowledge and training as primary concerns for barriers to EPM in their ICUs.

Several studies have discussed availability of personnel to perform the protocol as a potential barrier to EPM. ${ }^{[5,22,33]}$ In a prospective observational study at a single ICU, scarcity of PT staff was shown to be the most common reason that patients did not undergo EPM. ${ }^{[31]}$ Several studies have examined the impact of potential solutions for this barrier. Johns Hopkins Hospital conducted a quality improvement project examining the use of dedicated PT and OT staff in their ICU in conjunction with multidisciplinary education regarding EPM. The study found that with these interventions there was an improvement in delirium, physical rehabilitation, functional mobility, and a decrease in length of stay. ${ }^{[30]}$ In another meta-analysis, possible solutions to this barrier were explored among three different hospitals. ${ }^{[14]}$ One center found that conducting a pilot study illustrated to organizational leadership that EPM was safe and effective. This data was presented to hospital leadership and funding was granted to provide additional staffing needed for successful EPM implementation in the ICU. ${ }^{[14]}$ Another center utilized PT students to improve staffing numbers where available. ${ }^{[14]} \mathrm{Ul}-$ timately, appropriate multidisciplinary staffing is a common barrier to EPM. It remains a vital component of an effective EPM programs and should be addressed. ${ }^{[30]}$

Lack of knowledge and training has been identified as a primary barrier for many facilities attempting to implement EPM. ${ }^{[14]}$ Lack of knowledge and training may be complex and may include problems with communication among team

Published by Sciedu Press members, collaborating times for care, and sedation medication schedules. ${ }^{[8]}$ Team members may also express difficulty with mobilizing the patient on life sustaining equipment. ${ }^{[5,22,33]}$ The Johns Hopkins quality improvement study previously noted incorporated multidisciplinary team education as a component of their EPM program and did see a positive impact on patient outcomes as previously noted. ${ }^{[30]}$ Another quality improvement project was conducted in an urban public hospital ICU in South Australia involving staff education and identification of perceived barriers to mobilization. ${ }^{[34]}$ This project concluded that education and interventions to improve leadership around EPM made a significant impact on patients' maximum level of mobility achieved but had no significant impact on other patient outcomes. ${ }^{[34]}$ This illustrates the importance of addressing other barriers identified such as sedation of the patient and staffing issues. ${ }^{[34]}$ Multidisciplinary team education, a "staff champion" or EPM liaison may be helpful in promoting ongoing education across disciplines regarding EPM specifically. ${ }^{[14]}$ Creating a multidisciplinary protocol can be highly effective in standardizing care surrounding EPM but must be accompanied by ongoing training and education. ${ }^{[14]}$ Ongoing staff education across disciplines that includes compliance checks and site visits by designated personnel is vital to ongoing success. ${ }^{[14]}$ Ultimately, the literature illustrates the importance of education of staff as a vital component of EPM success. ${ }^{[14]}$

Lack of leadership is one other barrier that has been identified in the literature as a potential barrier. ${ }^{[14]}$ The Australian quality improvement study mentioned previously examined the effects of interventions to promote leadership with EPM and observed significant impact on patients' maximum mobility level but did not see significant impact on other patient outcomes. ${ }^{[34]}$ This again demonstrates the importance of addressing leadership needs of EPM but also emphasizes that this intervention alone will not lead to ideal outcomes. ${ }^{[34]}$ Another research group undertook an educational intervention and staff survey to attempt to change staff perceptions around EPM in their surgical ICU. ${ }^{[35]}$ Utilizing the PlanDo-Study-Act model, they found that operational support contributed to improved attitudes of staff around EPM. ${ }^{[35]}$ Essential elements to addressing this barrier include recruitment of leadership from all involved disciplines. ${ }^{[14]}$ This would typically include leadership from assistive personnel, PT, OT, nursing, and medical providers. A physician leader may lead the process of quality improvement within the interdisciplinary team. ${ }^{[14]}$

Lack of physician referral for PT/OT has been identified as an ongoing barrier to EPM in the literature. ${ }^{[14]}$ This barrier can be easily remedied by creating an automatic medical provider order on admission and having a focused mobility 
leader or liaison audit for the presence of this order on the day of admission. ${ }^{[14]}$ The use of daily mobility assessment and treatment where appropriate has also been found to be effective. ${ }^{[14]}$

Sedation has been established as a significant barrier to EPM. ${ }^{[36]}$ In a prospective chart audit, one group of researchers identified barriers to EPM in their medical-surgical tertiary ICU. ${ }^{[37]}$ They concluded that mobilization was safe and effective but met with several barriers including sedation, vascular catheter location, and procedure interference. ${ }^{[37]}$ This project illustrated that improved sedation management was necessary to improve EPM at this site. ${ }^{[37]}$ Sedation can be safely minimized with activity. ${ }^{[36,38]}$ In one pre-post study, increased ambulation was observed in patients who had not received sedation. ${ }^{[19]}$ Another prospective cohort study showed that patients took longer to consult with a physical therapist when they were receiving continuous sedation. ${ }^{[23,26]}$ Methods to reduce this barrier include the use of bolus sedation in the place of infusion sedation and avoidance of benzodiazepines except where indicated for seizures or other qualifying disorders. ${ }^{[14]}$

\subsection{Synthesis of literature findings}

EPM is a safe and effective treatment measure that may significantly impact patient outcomes when appropriate barriers to its implementation are addressed. ${ }^{[2]}$ EPM reduces cost of healthcare, duration of mechanical ventilation, length of stay, and improves functional mobility at discharge as well as quality of life at discharge. ${ }^{[8]}$ The use of protocols and standardized care approaches around EPM is becoming commonplace across ICU's nationwide. ${ }^{[2]}$ This has led to emerging research around the barriers that are commonly experienced in implementing EPM protocols. Barriers that have emerged in this research include lack of dedicated leadership for EPM, lack of staffing and equipment, lack of physician referrals for PT, over-sedation, and lack of knowledge and training. ${ }^{\text {[2] }}$ These barriers may be addressed in various ways. Most importantly, being aware of these barriers and finding system based solutions may have a positive impact on successful EPM implementation and ultimately patient outcomes. ${ }^{[2]}$

\section{MethodS}

This quality improvement project utilized a cross-sectional descriptive correlational design to examine the relationship among the described variables. A collaborative effort among a multidisciplinary team was utilized to develop the project survey tool which consisted of a pre- and post-survey designed to assess KSAs. The survey tool included the following: part 1, description of the project and consent; part 2, demographic questions; part 3, knowledge questions; part 4, skills questions; part 5, attitude questions; part 6, reported compliance based on discipline questions. The questions were designed using a five-point Likert scale, closed-ended questions (yes/no, true/false, select all that apply), and open ended questions. Content validity was established using an expert panel of three individuals which yielded a content validity index of 1.0. Institutional Review Board (IRB) exemption status from both Touro University Nevada and the host site was obtained. Approval from the stakeholders and Chief Nursing Officer and Chief Executive Officer at the project site was obtained prior to the implementation of the project. Two live training sessions that were identical in nature were held to provide education to participants and allow for diversity of schedules. The paper pre-test was administered prior to the educational intervention. The paper post-test was administered after the educational intervention. Data was then input into Survey Monkeyand $($ IBM's Sta-

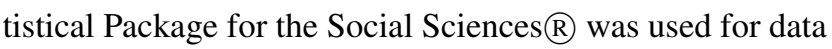
analysis.

Participants were invited via email, flyers, and word of mouth from management services, the unit director, and the unit educator to attend an educational seminar. Two separate dates were provided to allow for diversity in scheduling. Participants targeted for recruitment included registered nurses' (RN's), PT's, OT's, RT's, and licensed medical providers at the host site. Incentives included lunch served at the meeting and a free iPad giveaway at each session. Funding for this was obtained through the Mentored Student Research Grant Award at Touro University Nevada.

The project sample consisted of RN's, PT's, OT's, RT's and licensed providers at the host site. Data was collected on a total of 26 participants.

Frequency distributions were the primary method of data analysis for this project. Descriptive statistics were included to describe population characteristics and check for violation of assumptions.

\section{RESULTS}

Twenty-three $(85.2 \%)$ of the 26 participants in this project identified as RN's, three (11.1\%) identified as PT's, and one participant $(3.7 \%)$ failed to respond to the question. One (3.7\%) of the participants failed to respond to the question regarding years in current position. Eleven $(40.7 \%)$ reported less than one year of experience, four $(14.8 \%)$ reported at least one year but less than three years, three $(11.1 \%)$ reported at least three years but less than five years, six $(22.2 \%)$ reported at least five years but less than 10 years, and two $(7.4 \%)$ reported 10 years or more. Regarding working in a previous position with a similar protocol, one (3.7\%) partic- 
ipant failed to respond to the question, seven (25.9\%) have worked in a previous position with a similar protocol, and nine $(70.4 \%)$ had not.

To answer the first project question, frequencies of scores on the survey were compared pre-test and post-test on the knowledge variable. The greatest number of participants scored a $70 \%$ and $75 \%$, which had five $(21.7 \%)$ participants in each scoring range for the pre-test, respectively. For the post-test, the greatest number of participants scored an $85 \%$, which had 12 (52.2\%) participants in this range. The average score among all participants increased from $75.9 \%$ to $83.2 \%$ from pre-test to post-test. To answer the second project question, the greatest number of participants indicated a $100 \%$, which had 10 (43.5\%) participants in this scoring range for the pre-test. For the post-test, the greatest number of participants also indicated $100 \%$, but this number increased from 10 to $13(56.5 \%)$ in this scoring range. The average score among all participants increased from $86.9 \%$ to $90.1 \%$ from pre-test to post-test.

To answer the third project question, the greatest number of participants indicated an $80 \%$, which had five $(21.7 \%)$ participants in this category on the pre-test. On the post-test, the greatest number of participants indicated both $72 \%$ and $91 \%$, which had 4 (17.4\%) in each of these categories, respectively. The average score among all participants increased from $71.9 \%$ to $74.4 \%$ from pre-test to post-test. Until further data is collected, data analysis on the fourth project question has been deferred.

\section{Discussion}

While the benefits of early mobilization in the intensive care unit have been well documented in recent years, many intensive care units are unable to effectively integrate EPM into their daily practice. This project determined that an educational module addressing perceived barriers to compliance of the EPM protocol was effective in increasing the knowledge level of the protocol at the project site. The educational intervention also increased reported skill in implementing the protocol at the project site. Lastly, the educational intervention improved the attitudes towards the protocol at the project site. Due to lack of representation of all disciplines, the project was unable to determine whether there is a difference in the reported likelihood to comply among various disciplines to the EPM, and is a limitation of this project. This project, through educational intervention using a multidisciplinary approach, can be helpful to ICU staff and administrators in underserved areas in addressing barriers to compliance with the EPM and other best practice protocols, ultimately promoting patient safety and quality patient care.

\section{Conclusion}

Implications denoted from this project for the ICU multidisciplinary healthcare team include the following: (a) EPM is a safe and effective intervention in intubated patients upon stabilization of physiologic alterations; (b) physiologic stabilization often occurs prior to liberation from mechanical ventilation and low-dose vasopressor infusion; (c) a multidisciplinary approach leads to improved success with EPM; (d) avoidance of benzodiazepine administration in critically ill patients except in the case of seizures, DT's, or anxiety can reduce delirium and improve mobilization efforts from the patient; (e) minimization or even eliminate sedation in intubated patients and in those where this is not possible perform daily sedation vacations.

A multidisciplinary approach to overcoming barriers can lead to improved compliance with an EPM protocol and support early rehabilitation to minimize functional decline of patients. Recommendations to improve associated outcomes include: (a) assign an EPM leader; (b) obtain proper dedicated equipment for each unit where the protocol is implemented such as a front-wheel walker and wheelchair; (c) acknowledge staff to patient ratios can have a significant impact on feasibility of EPM; (d) utilize non-licensed assistive personnel; (e) mandatory computerized entry of mobilization orders and mobilization progression; and (f) a multidisciplinary team collaboration effort.

This project aligns with the literature regarding the difficulty of establishing quality improvement initiatives in underserved areas, which may be due to limited access to resources for successful implementation of an EPM protocol. Further studies need to be conducted to establish meaningful methods to promote compliance in these areas. This project demonstrated an increase in knowledge level, skill in implementation, and attitudes toward the protocol after a targeted educational intervention. Routine educational intervention regarding EPM and multidisciplinary involvement in EPM initiatives may serve to improve KSAs related to this best practice protocol.

\section{ACKNOWLEDGeMENTS}

Funding for this quality improvement project was received through the Touro University Nevada Mentored Student Research Grant Award. This project team would also like to acknowledge Justina Selim for her contributions as a research assistant.

\section{CONFlicts OF INTEREST Disclosure}

The authors declare that there is no conflict of interest. 


\section{REFERENCES}

[1] Zomorodi M, Topley D, McAnaw M. Developing a mobility protocol for early mobilization of patients in a surgical/trauma ICU. Crit Care Res Pract. 2012; 2012: 964547.

[2] Retrieved November 1, 2018. Last updated 2018. Available from: https : //www-uptodate-com.1b-proxy2.touro.edu/cont ents/post-intensive-care-syndrome-pics?search=ear 1y\%20mobilization\&source=search_result\&selectedTit $l e=2 \sim 150 \& u s a g e \_t y p e=d e f a u l t \& d i s p l a y \_r a n k=2$

[3] Retrieved November 1, 2018. Last updated 2018. Available from: http://www. sccm.org/ICULiberation/ABCDEF-Bundl es/Early-Mobility

[4] Leditschke IA, Green M, Irvine J, et al. What are the barriers to mobilizing intensive care patients? Cardiopulm Phys Ther J. 2012; 23: 26-29.

[5] Dammeyer J, Dickinson S, Packard D, et al. Building a protocol to guide mobility in the ICU. Crit Care Nurs Q. 2013; 36(1): 37-49. https : //doi.org/10.1097/CNQ.0b013e3182750acd

[6] Dang SL. ABCDEs of ICU: Early mobility. Crit Care Nurs Q. 2013; 36(2): 163-168. https://doi.org/10.1097/CNQ.0b013e3182 $83 \mathrm{cf} 45$

[7] Bailey P, Thomsen G, Spukler V, et al. Early activity is feasible and safe in respiratory failure patients. Critical Care Medicine. 2007; 35(1): 139-145. https://doi.org/10.1097/01.CCM.0000251 130.69568 .87

[8] Parker A, Needham D. The Importance of early rehabilitation and mobility in the ICU. Society of Critical Care Medicine. 2013.

[9] Girard TD, Alhazzani W, Kress JP, et al. ATS/CHEST Ad Hoc Committee on Liberation from Mechanical Ventilation in Adults. An Official American Thoracic Society/American College of Chest Physicians Clinical Practice Guideline: Liberation from Mechanical Ventilation in Critically Ill Adults. Rehabilitation Protocols, Ventilator Liberation Protocols, and Cuff Leak Tests. Am J Respir Crit Care Med. 2017 Jan 1; 195(1): 120-133.

[10] Martin UJ, Hincapie L, Nimchuk M, et al. Impact of whole-body rehabilitation in patients receiving chronic mechanical ventilation. Crit Care Med. 2005; 33(10): 2259-2265. https ://doi.org/10 $.1097 / 01$. CCM.0000181730.02238.9B

[11] Mendez-Tellez PA, Nusr R, Feldman D, et al. Early Physical Rehabilitation in the ICU: A Review for the Neurohospitalist. Neurohospitalist. 2012; 2(3): 96-105. https : //doi.org/10.1177/194187 4412447631

[12] Montagnani G, Vagheggini G, Panait Vlad E, et al. Use of the Functional Independence Measure in people for whom weaning from mechanical ventilation is difficult. Phys Ther. 2011; 91(7): 11091115. https ://doi.org/10.2522/ptj . 20100369

[13] Schweickert WD, Pohlman MC, Pohlman AS, et al. Early physical and occupational therapy in mechanically ventilated, critically ill patients: a randomised controlled trial. Lancet. 2009; 373(9678): 1874 1882. https://doi.org/10.1016/S0140-6736(09)60658-9

[14] Engel HJ, Needham DM, Morris PE, et al. ICU early mobilization: from recommendation to implementation at three medical centers. Crit Care Med. 2013 Sep; 41(9 Suppl 1): S69-80.

[15] Hermans G, Van Mechelen H, Clerckx B, et al. Acute outcomes and 1-year mortality of intensive care unit-acquired weakness. A cohort study and propensity-matched analysis. Am J Respir Crit Care Med. 2014 Aug 15; 190(4): 410-20.

[16] De Jonghe B, Sharshar T, Lefaucheur JP, et al. Groupe de Réflexion et d'Etude des Neuromyopathies en Réanimation. Paresis acquired in the intensive care unit: a prospective multicenter study. JAMA. 2002 Dec $11 ; 288(22)$ : 2859-67.
[17] Griffiths J, Hatch RA, Bishop J, et al. An exploration of social and economic outcome and associated health-related quality of life after critical illness in general intensive care unit survivors: a 12-month follow-up study. Crit Care. 2013 May 28; 17(3): R100.

[18] Parry SM, Nydahl P, Needham DM. Implementing early physical rehabilitation and mobilisation in the ICU: institutional, clinician, and patient considerations.Intensive Care Med. 2018 Apr; 44(4): 470-473. https ://doi.org/10.1007/s00134-017-4908-8

[19] Dong ZH, Yu BX, Sun YB, et al. Effects of early rehabilitation therapy on patients with mechanical ventilation. World J Emerg Med. 2014; 5(1): 48-52. https://doi.org/10.5847/wjem.j.issn. 1920-8642.2014.01.008

[20] Hunter A, Johnson L, Coustasse A. Reduction of intensive care unit length of stay: the case of early mobilization. Health Care Manag. 2014; 33(2): 128-135.

[21] Koukourikos K, Tsaloglidou A, Kourkouta L. Muscle atrophy in intensive care unit patients. Acta Inform Med. 2014; 22(6): 406-410. https://doi.org/10.5455/aim.2014.22.406-410

[22] Pohlman MC, Schweickert WD, Pohlman AS, et al. Feasibility of physical and occupational therapy beginning from initiation of mechanical ventilation. Crit Care Med. 2010; 38(11): 2089-2094. https ://doi.org/10.1097/CCM.0b013e3181f270c3

[23] Schweickert WD, Kress JP. Implementing early mobilization interventions in mechanically ventilated patients in the ICU. Chest. 2011; 140(6): 1612-1617. https ://doi.org/10.1378/chest.10-282

[24] Schujmann DS, Lunardi AC, Fu C. Progressive mobility program and technology to increase the level of physical activity and its benefits in respiratory, muscular system, and functionality of ICU patients: study protocol for a randomized controlled trial. Trials. 2018 May 10; 19(1): 274. https ://doi .org/10.1186/s13063-018-2641-4

[25] Girard TD, Kress JP, Fuchs BD, et al. Efficacy and safety of a paired sedation and ventilator weaning protocol for mechanically ventilated patients in intensive care (Awakening and Breathing Controlled trial): a randomised controlled trial. Lancet. 2008; 371(9607): 126-134. https ://doi.org/10.1016/S0140-6736(08)60105-1

[26] Schaller SJ, Anstey M, Blobner M, et al. International Early SOMSguided Mobilization Research Initiative. Early, goal-directed mobilisation in the surgical intensive care unit: a randomised controlled trial. Lancet. 2016 Oct 1; 388(10052): 1377-1388.

[27] Morris PE, Goad A, Thompson C, et al. Early intensive care unit mobility therapy in the treatment of acute respiratory failure. Crit Care Med. 2008 Aug; 36(8): 2238-43.

[28] Parker A, Needham D. The Importance of early rehabilitation and mobility in the ICU. Society of Critical Care Medicine. 2013.

[29] Hopkins RO, Spuhler VJ, Thomsen GE. Transforming ICU culture to facilitate early mobility. Crit Care Clin. 2007; 23: 81-96. https://doi.org/10.1016/j.ccc.2006.11.004

[30] Needham DM, Korupolu R. Rehabilitation quality improvement in an intensive care unit setting: implementation of a quality improvement model. Top Stroke Rehabil. 2010; 17: 271-281. https : //doi.org/10.1310/tsr1704-271

[31] Zanni JM, Korupolu R, Fan E, et al. Rehabilitation therapy and outcomes in acute respiratory failure: an observational pilot project. $\mathbf{J}$ Crit Care. 2010; 25: 254-262. https ://doi.org/10.1016/j.jc rc. 2009.10.010

[32] Mendez-Tellez PA, Needham DM. Early physical rehabilitation in the ICU and ventilator liberation. Respir Care. 2012; 57: 1663-1669. https://doi.org/10.4187/respcare.01931

[33] Adler J, Malone D. Early mobilization in the intensive care unit: a systematic review. Cardiopulm Phys Ther J. 2012; 23(1): 5-13. https://doi.org/10.1097/01823246-201223010-00002 
[34] Dafoe S, Chapman MJ, Edwards S, et al. Overcoming barriers to the mobilisation of patients in an intensive care unit. Anaesth Intensive Care. 2015 Nov; 43(6): 719-27.

[35] Castro E, Turcinovic M, Platz J, et al. Early Mobilization: Changing the Mindset. Crit Care Nurse. 2015 Aug; 35(4): e1-5; quiz e6.

[36] Witcher R, Stoerger L, Dzierba AL, et al. Effect of early mobilization on sedation practices in the neurosciences intensive care unit: A preimplementation and postimplementation evaluation. J Crit Care.
2015; 30(2): 344-347. https ://doi.org/10.1016/j .jcrc. 201 4.12 .003

[37] Leditschke IA, Green M, Irvine J, et al. What are the barriers to mobilizing intensive care patients? Cardiopulm Phys Ther J. 2012 Mar; 23(1): 26-9.

[38] TEAM Study Investigators, Hodgson C, Bellomo R, et al. Early mobilization and recovery in mechanically ventilated patients in the ICU: a bi-national, multi-centre, prospective cohort study. Crit Care. 2015 Feb 26; 19: 81. 